\title{
Trabecular Meshwork Proliferation
}

National Cancer Institute

\section{Source}

National Cancer Institute. Trabecular Meshwork Proliferation. NCI Thesaurus. Code C161558.

Activation of abnormal cell growth within the trabecular meshwork of the eye, usually due to injury. 